\title{
A Participatory Approach for Game Design to Support the Learning and Communication of Autistic Children
}

\author{
Thiago Porcino ${ }^{1,}$ Daniela Trevisan ${ }^{1}$, Esteban Clua ${ }^{1}$, Marcos Rodrigues ${ }^{1}$ and \\ Danilo Barbosa ${ }^{1}$ \\ ${ }^{1}$ Federal Fluminense University, Niterói, Rio de Janeiro, Brazil \\ \{thiagomp, daniela, esteban\} @ic.uff.br \\ \{marcosrodrigues, danilo\}@id.uff.br
}

\begin{abstract}
In this work we propose to apply a participatory design process for developing mobile games focused on learning and communication of autistic children. This study employs a game to help people with autism and describes the complete design process used in this research. As a result of the design process was possible to note the necessity to allow high customization and personalization of digital activities in order to promote the user engagement and gameplay ability. Moreover, more details are provided about the developed game. Two customized interactive activities were developed: Questions \& Answers and the Emotional Thermometer that were evaluated with therapists, autistic children and HCI specialists. Finally, it is described the importance of therapists in game design process and the requirements for redesigning the application interface.
\end{abstract}

Keywords: Autism, children, learning, communication, mobile, participatory design, game.

\section{Introduction}

Autism is a general term for people who have one or more brain disorders that affects social interaction with different degrees: verbal and nonverbal communication, interests and behaviors. [1]

Autistic Spectrum Disorder (ASD) is a global development disorder that affects 1 in 68 children in the U.S., and may even be considered to be approaching epidemic status [2]. Although a significant portion of the population is affected, a great deal of myth and misinformation is associated with this disorder [1]. As a result, these children face a scenario that is not only complicated due to their symptoms but worsened by a lack of comprehension and support.

It has been proven that many of the negative effects of autism can be minimized when treatment is initiated early [10]; therefore, appropriate tools are essential for the cognitive development of children with ASD. However, these tools should consider certain specific characteristics of the children in order to be effective projected. 
Although each case is unique, individuals with this disease share some limitations, which are not always easy to define. For example, difficulties with generalizing and attention to detail make it difficult to find appropriate pedagogical activities for children with ASD, as associating designs and other representations with their real counterparts are too complicated. Many of these children are unable to communicate correctly, and some reach adulthood without the ability to communicate verbally.

Children with ASD appreciate the safe and replicable environment of computer tools $[1,7]$. However, as pointed in the Related Works session, there is a lack of tools and games that are guaranteed to be flexible and scalable, with experiences that are integrated, free, and, above all, accessible.

Accordingly, the contribution of this work can be summarized in two fold: the design process applied to develop a mobile game in order to assist parents and teachers in teaching these children and moving them towards a potentially independent life, while providing tools to facilitate communication; and the application itself. This application uses the benefits of devices such as smartphones and tablet PCs in order to allow more concrete experiences. This differs from the majority of software available on the market because it allows a high degree of customization and integration. This study is the first step in a participatory design process based on an initial prototype, which in turn was guided by the studies of the characteristics of autistic children.

\section{Related Works}

There are various applications for mobile devices that seek to facilitate communication and stimulate learning and cognitive development in people with autism. However, few integrate these functionalities in a cohesive and personalized environment. SCAI Autismo, an augmentative and alternative communication tool (AAC) $[11,21]$ based on PECS, shows cards containing an image and text on the screen. The cards include "I," which contains an image that may be personalized with a photo, affirmative and negative, and desires like drinking water, sleeping, and eating dinner. They may be selected sequentially, reproducing a pre-recorded audio, so that the child can express a desire. TalkinPictures [24], also based on PECS, is a commercial application that has categorical hierarchies of cards and permits a greater degree of personalization, using a text-to-speech solution in order to include new options added by the user. meaVOX [25], which was developed by the Universidade Federal Fluminense, is similar to TalkinPictures, but instead of using text-to-speech, it permits sounds to be recorded and associated with the cards, and uses a desktop program to configure the application data. The game "What's the Word" [26] (4 pictures 1 word) not only aids in literacy but also encourages the ability to generalize. Each challenge includes four separate figures. The player must discover the common element between them and choose a group of scrambled letters that form the correct word.

In fact, none of these games permits the challenges to be customized, and players are restricted to the options offered by the developer upon installation of the program or through in-app purchases.

\section{Design Process}


In order to develop an appropriate tool to attend the needs of therapists, parents and children, we establish a close communication with the Center of Optimization for Rehabilitation for Autists (CORA). It is a non-profit entity that helps dozens of families. Currently, CORA has 5 therapists: one psychologist, one speech therapist, one music therapist, and two educational psychologists. At the moment of this research, approximately 45 patients were being treated, including children and adolescents from 5 to 16 years, being 14 of them females. There is a study and activity room and the main hall, where group activities are performed. In the kitchen, there is a sink and a table for lunch and breakfast. There is no equipment that would put patients at risk. The study room has 5 individual tables. This is the room where the learning activities are conducted. When the children have successfully performed tasks, they can visit the game room and participate in their favorite activities as a reward. The game room has a rubber floor and some toys such as balls and dolls. The main room is the place where the children interact as a group through games or toys, such as interviewer and interviewee, where a child is the interviewer and asks questions to the others sitting at the table.

\subsection{Defining context of use}

Analysis of the context in which the application would be used included interviews, questionnaires, and examination of footage taken previously of routine activities that were conducted at the beginning of the study. The questionnaire used has been made available in Appendix A [27].

The responses to the closed questions revealed that although the therapists were between 30 and 65 years of age, they frequently used the mobile devices with success, and considered them useful tools for therapy, because patients generally already have had contact with them and appreciate using this type of technology at home, mainly for electronic games. The majority of them are or were married, have at least one autistic child, and demonstrated great satisfaction with and commitment to their job at CORA. Only one is currently pursuing higher education; all the others have already concluded or are pursuing post-graduate education. Therefore, one may conclude that all are equally capable of providing valuable opinions on the needs of the patients, using the proposed application without much difficulty, and participating in the process of assessment while helping the child. They also indicated that the mobile devices, especially tablets, are appropriate for use both with patients and therapists, as was expected.

Using open questions, we noted that the activities most used to help patients learn are games involving the association of concepts, for example, pairing figures, as well as activities that involve music or other sound elements. The exercises are usually adjusted for each child according to his or her interests. We also observed that the children enjoy watching their favorite television programs and using computers, which was mainly seen when they always responded to particular input in the same manner. As for the association of concepts, the importance of realism was highlighted, that is, concepts must be represented by actual images and not fictitious ones because autistic children have difficulty understanding symbols. It was also suggested that the application should address issues related to speech, reading and social issues.

During the interviews, besides repeating the points raised in the questionnaire, it was noted that some therapists already use applications on their mobile devices (not always especially for autistic children) to assist in therapy. However, a single 
application may not be appropriate for multiple children, and various applications are needed to assist different patients.

The filmed activities were mostly group games focused on movement, encouraging fine motor development. It noted that during group sessions and more recreational and physical activities, the children were very extroverted and cooperative. However, they were less engaged in individual activities focused on learning, such as color association.

Accordingly, it was possible to confirm many of the premises obtained in prior studies as well help acquire new knowledge. Three types of people were identified from the data [4]: the therapists, who had very similar backgrounds, and the other two representing typical children who attend CORA. These are the target user profiles for the application: autistic children up to 10 years of age who mainly have communication problems. A scenario describing routine activities was also prepared, focusing on problems that might arise in these circumstances. This data guided the subsequent stages, and was more definitively prepared through the participatory design process. Factors such as the need for concrete elements, feedback sound, repeatability, appropriateness of the activity for each child in terms of both the area to be trained and individual interests, and focus on the association of concepts was strongly addressed during the application's development

\subsection{Participatory Design}

Participatory design is an approach that attempts to include the interested parties in the design process, i.e., all those directly or indirectly involved with the product [16]. In order to immerse the autistic children in the participatory design process, the Interface Design Experience for the Autism Spectrum (IDEAS) method was applied. The goal of this method is to include autistic children along with a team of specialists in the activities involved in designing a system, in a participatory design process [16].

The participatory design technique was used to guide development, ensuring that the proposed solutions meet the real needs of the end users in addition to encouraging a feeling of ownership of the application. The procedures were conducted together with two therapists jointly with the occasional cooperation of others, and comprised three meetings of about 1 hour divided into the following stages:

1. Review of the changes planned in the previous meeting;

2. Presentation of the changes implemented;

3. Validation and criticism of changes

4. Creation of new ideas

5. Definition of subsequent changes

Changes are defined as any modification to the current state of the application, whether an alternative of existing functions or creation of new ones. Between the meetings, the adjustments arising from the critiques and new modifications were implemented.

\section{Design Solution}


At the conclusion of the participatory design process, two main digital activities were performed: Questions and Answers and Card Keyboard, with the sub-game Emotional Thermometer. Below are given definitions for some terms that are referenced throughout the following sections:

\section{Card}

The activities are based on this component. It has a name and may have an image, text, and audio, all of which are optional.

\section{Tutor}

The therapist, father, or mother of the child, responsible for accompanying him or her during the usage of the application, answering questions, stimulating and personalizing the experience.

The game Questions and Answers (Fig. 1) was planned as a tool for assisting in learning, and comprises a series of questions. Each card includes a main card, represented by a large image that should be associated with one of the available options (also cards), which can be represented by a smaller image or text (if the child can read). Both the question and its answers may contain an audio instructing the user on how it should be answered, or refining the referenced concept when it is selected, respectively. If the user selects the correct option, the system provides positive feedback with a sound, and shows a dialogue box with the word "Correct!". The background is then changed for the next question. If the answer is incorrect, a different sound and the text "Incorrect" appears. The user may take as many attempts as he or she wishes, and return to the previous questions. The next question can only be reached by successfully answering the current one. In order to finish the game, the player must answer all the questions.

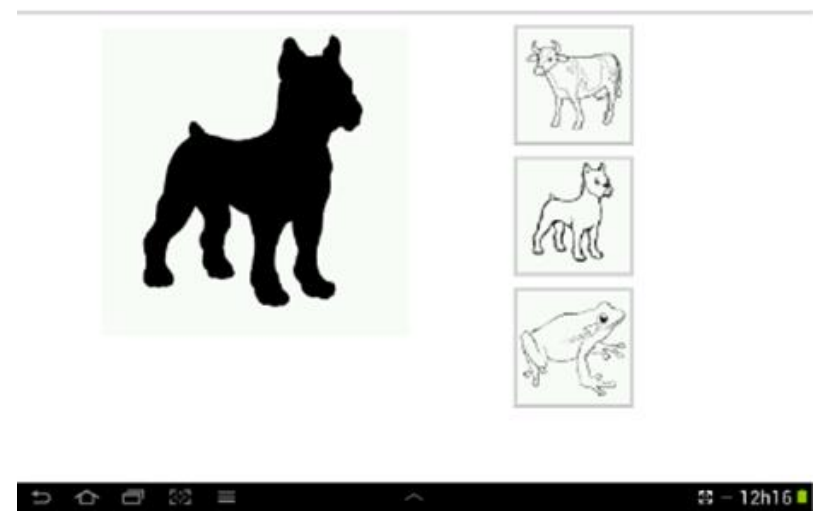

Fig. 1. Questions and Answers game interface.

Two alternative communication games were developed. The first one is the Emotional Thermometer (Fig. 2), based on a solution proposed by Attwood [3, p. 155] [23, p.131], is a horizontal sequence of photographs of the faces of children with different facial expressions. These are organized in a range of emotions from irritated to bored to very happy, using a slider component (Fig. 3) underneath so that the user may indicate the emotion that most closely matches his or her own. This resource is available in the game Questions and Answers, and can be accessed by pressing the screen for more than 500 milliseconds; in this way, if the user wants to stop the activity, the tutor may activate the thermometer or request that the child explain how he or she is feeling. 


\section{1을}

Fig. 2. Emotional Thermometer slider.

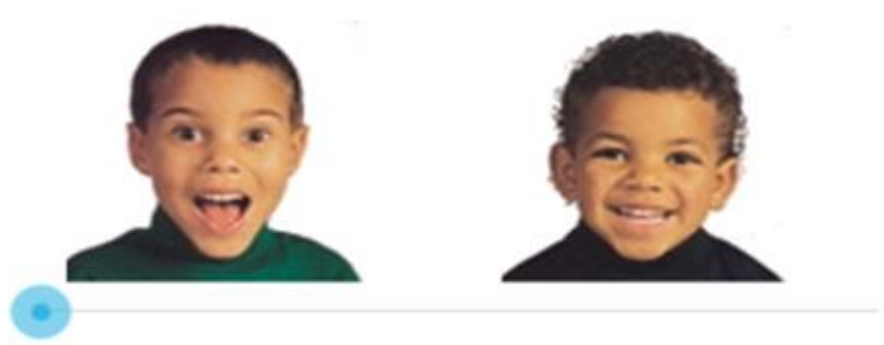

Fig. 3. Slider for emotional calibration.

The other communication game is the Card Keyboard (Fig. 4) based on the Picture Exchange Communication System (PECS) [23]. It comprises different cards that contain an image and a sound, divided into three categories:

Who?: Subject who performs the action. e.g., I, you.

Action: Action. e.g., want, do not want.

Which one?: Object to which the action is applied. e.g., food, bath.

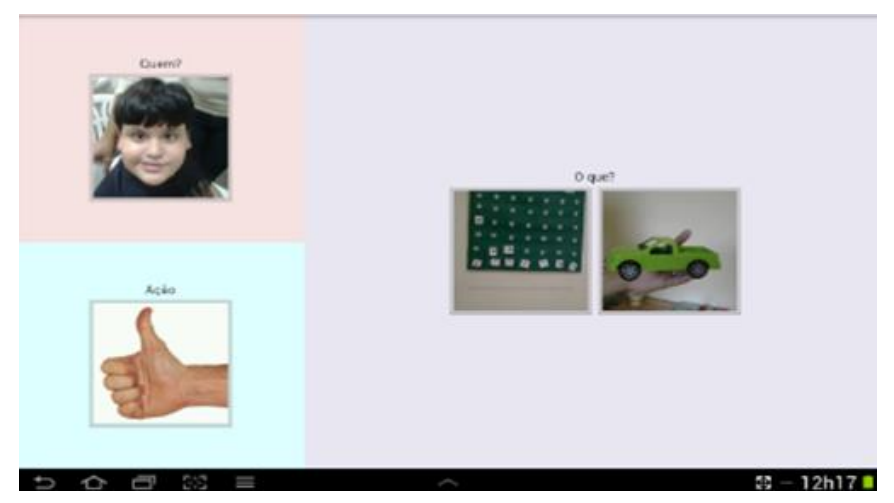

Fig. 4. Card Keyboard game interface.

The main objective is to make the children use the cards in order to communicate what they want or do not want to do. In this manner, they can avoid frustration and the potential of attacks resulting from not knowing how to verbalize their wishes. This activity has its own area, as it must be accessed when there is a clear purpose, and not as the result of actions in other parts of the application.

In order to satisfy the requirement to provide sound feedback, taking advantage of the sensory interests of children with ASD in a general manner, images, text, and sounds were combined in the game, and touch was used as a way of interacting, thereby taking advantage of the benefits for learning and the developments achieved using this 
type of interface $[13,17]$. The touch interface is more concrete than using a mouse; touching is a natural gesture with immediate feedback, which the child already knows. However, it was decided during the design meetings with the therapists that it would be more useful to add the possibility of using drag-and-drop in the game Questions and Answers in order to strengthen the association between the principal card and the option selected. This type of interaction is also more appropriate for children as pointed in [6]. This is represented visually by a colored line linking the initial touch point to the current finger position, and also adds a colored frame around the principal card as well as the option underneath the user's finger, if applicable.

As a relevant result from the participatory design sessions was possible to identity the need to have facilities for customization of game objects. In order to allow customization, all the cards may be modified by the tutor using the device's microphone, camera, and image gallery. In this way, the topics and options in Questions and Answers can be applied to the word that the child understands, incorporating subjects of interests and increasing engagement. Similarly, the Card Keyboard can represent the child's reality, for example, her or his own photo for the "I" card, and photos of favorite meals to represent for example "food." It was also decided that the order of questions should be modifiable to allow a gradual increase in the difficulty and grouping of related questions, as well as the order of the cards within each Card Keyboard category. The interface was kept as simple as possible, showing only the elements required for the current activity (which runs in full screen, without a notification bar), thereby removing any distractions. Initially, each tool had its own section, which included the option to start it (targeting the child) or to configure it (targeting the tutor). After the first day of use in the session, however, it became clear that more separation according to the user profile was necessary. Consequently, the start screen of the game has buttons to start each of the activities and button slightly farther away to enter the tutor interface (see Fig. 5).

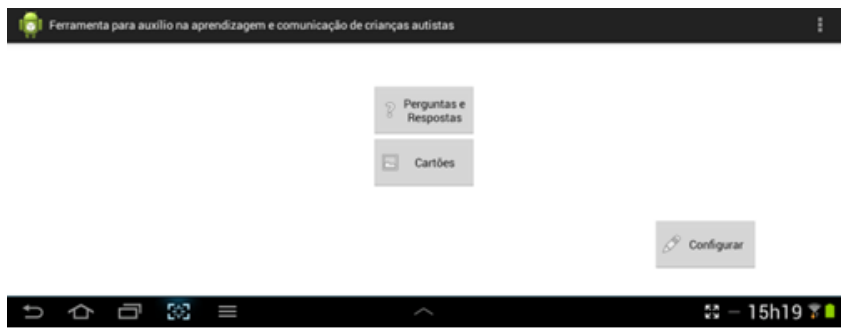

Fig. 5. Start game screen.

The screen elements always correlate to the various interactions, visually and through sounds, indicating the ability to select active selection and thereby providing feedback to the user. Repeatability was achieved, allowing the user to return to any step of any activity.

\section{Evaluation}

The DECIDE framework [4], which defines the planning, execution and analysis of an Human Computer Interaction evaluation was used to establish the procedures and issues in assessing the proposed solution. The objectives, represented by questions to be answered at the end of the evaluation process, were:

- Is the child capable of understanding and independently using the game Questions and Answers until the end?

- Is the child capable of understanding the objective and successfully 
expressing himself using the alternative and amplified communication methods?

- When performing the proposed tasks, is the child capable of carrying out the required actions?

- Is s it possible to adjust the activities to the child's reality?

- Was the child experience with the game enjoyable?

Because the main users of the application will be autistic children, the majority of whom have little or no verbal capability, the only reliable way of obtaining the answers to these questions would be through usability and accessibility tests. Therefore, this method of evaluation was chosen, using a process filmed with the consent of both of the children's parents and respecting the appropriate ethical considerations.

The procedures to be executed were explained in a meeting with the parents and the therapists; any questions were answered, and the researchers' contact information was provided to ensure transparency. The game was also presented, so that they could approve its use by their children. The necessary equipment (a digital camera and an Android tablet) was reserved and prepared. Preparation of the tablet included extensive tests of the device's functions in order to ensure a fluid and bug-free experience; the activities were pre-configured with various questions and cards, following guidance from the therapists. Finally, the evaluation itself was performed. The methodology used for evaluation is thoroughly described below.

\subsection{Methodology}

For filming, 6 usage sessions addressing the explicit requirements of the TEACCH program were conducted [15]. The structure of the sessions was based on Benton et al. [8]. Each session involved a researcher filming and coordinating the procedures and answering any questions that might arise, a therapist acting as the tutor, and an autistic child age 3-10 who were experiencing her or his first contact with the game. The role of the therapist was to help the child, in the event of questions, to perform the actions necessary to complete the tasks, as well as to encourage him or her to continue. This also helped to address the need for routine once the child became accustomed to attending. For the same reason, the sessions were carried out at the CORA facilities, at the children's normally scheduled therapy time.

In order to address the difficulty of organization and sequencing, a list of tasks to be performed during the session was prepared. For all the items, this list was presented to the child at the beginning of the session and resumed after the conclusion of each task to promote concentration and the concept of the goal. The child was offered the opportunity to check off the items if they wished to create a feeling of participation and winning. The tasks performed include:

- Watch a video demonstrating the use of the game Questions and Answer.

- Play Questions and Answers.

- Watch a video demonstrating the use of Card Keyboard (nonverbal participants only).

- Use Card Keyboard (nonverbal participants only).

- Paint stars that correspond to a Likert satisfaction scale [14].

- As a reward for completing all the tasks, play any game installed on the tablet for 5 minutes (popular game options were offered).

At the end of the session, the therapist completed a questionnaire regarding the child interaction in order to facilitate more qualitative details in the evaluations. 
Table 1. Evaluated Children.

\begin{tabular}{|l|l|l|}
\hline Child Code & Age & Sex \\
\hline C1 & 5 & Male \\
\hline C2 & 10 & Female \\
\hline C3 & 3 & Male \\
\hline C4 & 4 & Male \\
\hline C5 & 6 & Male \\
\hline C6 & 10 & Male \\
\hline
\end{tabular}

\subsection{Method of Interaction Analysis}

To analyze the filmed content in the most objective manner possible, the DEVAN (Detailed Video Analysis) tool was used [5, 22]. Recently researchers have adapted the DEVAN tool to analyze the usability test videos for a game for children with Down syndrome entitled JECRIPE - Stimulation Game for Preschool Age Children with Down Syndrome [18]. This project used a table adapted for the children to measure elements such as fun and modified the table to contemplate the specific problems of interactions in this genetic disorder. In this method, various evaluators complete a form, and each record is composed of a code representing an interaction problem together with a timestamp for the moment of its occurrence. A second timestamp may be added if it lasts longer than a defined limit (in this case, four seconds). Because resources were limited, the same three non-specialist researchers involved in the study were used as evaluators. The table of codes was based on the one used by Macedo et al. in [18], with the inclusion of the ACX entry (exploratory action) representing autistic children's specific tendency to focus on details and lose focus on the context. The coding scheme used can be seen in Table 1.

Table 2. DEVAN method adapted for children with Down syndrome [18].

Code Description Definition

ACE Wrong An action does not belong in the correct sequence of Action actions.

An action is omitted from the sequence.

An action within a sequence is replaced by another action.

Actions within the sequence are performed in reversed order.

The user performs a wrong action unintentionally.

ACP Intentional The user knows that the action is wrong, but still Wrong performs this action only to have fun.

AJU Help

The user cannot proceed without help or the researcher has to intervene in order to prevent serious problems.

The user is helped to do some action.

ANT Dislike The user indicates disliking something.

CON Puzzled The user indicates not knowing how to proceed.

IMP Impatience The user shows impatience by clicking repeatedly on objects that respond slowly, or when it takes too much time to reach the desired goal.

PAS Passive The user stops playing and does not perform the expected action.

PEX Execution The user has physical problems during interaction Problem with the game.

The user has motor skill problem.

PPR Perception The user indicates not being able to hear or see Problem something clearly, not understanding how to 
proceed.

$\begin{array}{lll}\text { RAN } & \begin{array}{l}\text { Random } \\ \text { Actions }\end{array} & \text { The user performs random actions. } \\ \text { STP } & \begin{array}{l}\text { Scenario } \\ \text { Stopped }\end{array} & \text { The user stops the scenario before reaching the goal. } \\ \text { TED } & \begin{array}{l}\text { Bored } \\ \text { The user indicates being bored by sighing or } \\ \text { yawning. }\end{array}\end{array}$

\subsection{Analysis of Results}

When validating a method, it is very unlikely that different evaluators will agree exactly by giving identical results for all evaluation sessions. The any-two agreement method measures the extent of agreement on what problems the system contains for pairs of evaluators [12]. For each comparison the number of agreements, disagreements and single points were recorded, always considering the margin of 4 seconds to be counted as the same observation point. The script created to conduct this comparison is available in [20].

Due to the subjectivity of the analysis and the evaluators' lack of experience, the level of agreement reached was considered low for all of the comparisons. As can be seen in Table 2, the best any-two agreement was $21.29 \%$, with a total of 45 unique observed points, 50 deviations and only 26 agreements. In an extreme case, there were 210 deviations in the lowest any-two agreement comparison. This demonstrates a probable difference in the understanding of the codes, as the observed points were very concordant, leaving few unique points.

Table 3. Results of Any-Two Agreement.

\begin{tabular}{|c|c|c|c|c|c|}
\hline EvaluationA x Evaluation B & Any-Two & Agree & Disagree & Unique A & Unique B \\
\hline Evaluation 1 x Evaluation 2 & 13,00 & 36 & 210 & 23 & 8 \\
\hline Evaluation 1 x Evaluation 3 & 21,49 & 26 & 50 & 43 & 2 \\
\hline Evaluation 2 x Evaluation 3 & 13,11 & 16 & 79 & 17 & 10 \\
\hline
\end{tabular}

\section{Discussion}

Based upon the analysis of the results and observations made during the sessions, the following were the most problematic points found in the proposed solution:

- Occasional delayed response in the application.

- Buttons and images were too small; it was reported that some children do not see well.

- Child accidentally accessed the tutor (therapist) interface.

\section{In the game Questions and Answers:}

- Method for entering the Thermometer function is not intuitive and may be activated unintentionally.

- Child was not able to clearly determine when an answer was incorrect, and insisted on the error.

- Child did not feel rewarded upon completing the game.

- Child repeatedly attempted to drag-and-drop toward the option-question, an interaction not contained in the application, instead of the question-option.

- Child felt disoriented when question content changed. For example, when moving from questions about animals to questions about superheroes, or when changing from questions with just figures to questions with text. 
- Child felt bored or unmotivated during the questions.

- Child attempted to select the option but touches the wrong space and chooses the wrong selection by accident.

In the sub-game Emotional Thermometer:

- Child did not understand the purpose of the screen.

- Child did not know how to return to the previous environment.

In the game Card Keyboard:

- Child did not understand the purpose of this screen.

- Child did not follow instructions to select the cards.

- There are no feedback showing the end of the sentence.

- There may be many different options for the same concept. For example, there may be several cards for food, such as pasta, beans and hamburger. This makes comprehension and selection difficult.

At the beginning of the evaluations, questions regarding the proposed solution were prepared. It was hoped that the answers to these questions would be obtained after the procedures carried out. Based on the observations, the following conclusions were made:

Is the child capable of understanding and independently using the game Questions and Answers until its conclusion? The verbal children were highfunctioning autistic patients with a higher level of concentration and abstraction; consequently, they did not have significant problems performing any of the proposed tasks. However, nonverbal children or those developing their language skills required constant assistance from therapists. The problems that were observed cannot be attributed to lack of experience with the technology, as they were all familiar with tablets. Therefore, this objective was partially achieved, requiring us to address the existing problems so that it can be used with a broader range of patients on the autism spectrum.

Is the child capable of understanding the objective and successfully expressing himself or herself using the alternate and broadened communication methods? No alternative communication method was considered successful. This may be for various reasons: lack of a clear objective, i.e., a specific message to be codified for the child though the tool, with appropriate feedback demonstrating the meaning of the sentence; inexperience on the part of the therapists, who guided the activity, with this type of tool; difficulties inherent to the severity of the child's condition; and short time of use. Teaching a new form of communication is a slow and gradual process.

Upon performing the proposed tasks, is the child capable of performing the required actions? The children had some common problems when attempting to perform the actions that were required, generally in relation to the direction of movement or to the stopping point. However, most of the time they were able to carry out their intentions.

Can the activities be adjusted to the child's reality? Because only a short amount of preparation time was available before the session, and because the therapists were not familiar with the children's personal preferences, it was not possible to adjust the application to all of them. However, several series of questions in the game Questions and Answers were prepared between the session days and managed to arouse the interest of some patients. Furthermore, some of the nonverbal children demonstrated interest in the ability to take their own photograph and place it in Card Keyboard, although they did not understand the objective. 
Was the user experience agreeable for the child? The results of the Likert painted star scale are not reliable, since the children were encouraged by the therapists to paint the stars; additionally, they did not seem to understand the meaning of this activity. However, based on the videos and personal impressions, it was possible to perceive various reactions ranging from boredom and disinterest to curiosity and excitement, so much so that future studies may address the excited response to rewards.

Based on the problems raised and on the answers to the initial questions, a new design proposal was developed. This proposal will be submitted to the CORA therapists, beginning a new series of iterations in the participatory design process, the results of which may create new interesting observations.

The resulting game application will be made available at no cost and under an open source license in Google Play. Currently, its code and executable version are hosted at [20].

\section{Conclusion}

The experience acquired with this game design process shown that involvement of therapists was essential in the participatory design process, and the process of evaluation yielded valuable information that can only be achieved through actual use by end users, allowing a thorough analysis of the game interaction. However, due to the difficulty involved in the scope of the problem where extra care was required with the autistic users during the study in order to avoid any stress or discomfort from the proposed activities, further design iterations were not possible. Data was only collected at CORA place because of the difficulty in finding the user targeted who could participate in the study, but it would be helpful to find more users who could participate in future usability and accessibility tests.

The results obtained from the DEVAN method and subsequent analysis using the anytwo agreement yielded excessively low concordance. It would be interesting to investigate the reasons for these disparities, clarifying any confusion in defining the codes. The analysis was performed by three non-specialist evaluators, which can affect the results. An essential task for removing doubt in this process is including more evaluators, including specialists, and comparing the new results with the previous ones.

Several suggestions for redesigning the application interface were mentioned during the design process and evaluation stage. These changes will be implemented in a future project, following new participatory design iterations. These new versions should be used to conduct and analyze new usability tests, permitting comparison between this new data and the results from the previous interface.

\section{References}

1. Sole-Smith V. Common myths about autism spectrum, Meredith Coporation. 2014. Available at: http://www.parents.com/health/autism/myths-about-autism/.

2. Baio, J. Prevalence of autism spectrum disorder among children aged 8 years - autism and developmental disabilities monitoring network, 11 sites, United States, 2010. MMWR Surveill Summ 63 Suppl 2 (2014), 1-21.

3. Attwood, T. The complete guide to Asperger's syndrome / Tony Attwood, 1st hardcover ed. ed. Jessica Kingsley Publishers London ; Philadelphia, 2006. 
4. Preece, J., Rogers, Y., Sharp, H. (2002), Interaction Design: Beyond Human-Computer Interaction, New York: Wiley

5. Barendregt, W., and Bekker, M. M. Developing a coding scheme for detecting usability and fun problems in computer games for young children. Behav Res Methods 38, 3 (2006), $382-9$.

6. Barendregt, W., and Bekker, M. M. Children may expect drag-and-drop instead of pointand-click. In CHI '11 Extended Abstracts on Human Factors in Computing Systems, CHI EA '11, ACM (New York, NY, USA, 2011), 1297-1302.

7. Barry, M., and Pitt, I. Interaction design: A multidimensional approach for learners with autism. In Proceedings of the 2006 Conference on Interaction Design and Children, IDC '06, ACM (New York, NY, USA, 2006), 33-36.

8. Benton, L., Johnson, H., Ashwin, E., Brosnan, M., and Grawemeyer, B. Developing ideas: supporting children with autism within a participatory design team. In CHI ' 12 Proceedings of the SIGCHI Conference on Human Factors in Computing Systems. Association for Computing Machinery (ACM), New York, 2012, 2599-2608.

9. Bondy, A., Hortonm, C., and A., O. The picture exchange communication system: Helping individuals gain functional communication. Autism Advocate 3 (2010), 21-24.

10. Corsello, C. M. Early intervention in autism. Infants \& Young Children 18, 2 (2005), 7485 .

11. Grigis, D., and Lazzari, M. Augmentative and alternative communication on tablet to help persons with severe disabilities. In Proceedings of the Biannual Conference of the Italian Chapter of SIGCHI, CHItaly '13, ACM (New York, NY, USA, 2013), 17:1-17:4.

12. Hertzum, M., Interaction, C. F. H., and Jacobsen, N. E. The evaluator effect: a chilling fact about usability evaluation methods. Int. Journal of Human-Computer Interaction, 2001.

13. Hourcade, J. P., Bullock-Rest, N. E., and Hansen, T. E. Multitouch tablet applications and activities to enhance the social skills of children with autism spectrum disorders. Personal Ubiquitous Comput. 16, 2 (Feb. 2012), 157-168.

14. Jamieson, S., et al. Likert scales: how to (ab) use them. Medical education 38, 12 (2004), $1217-1218$

15. Mesibov, G. B., Shea, V., and Schopler, E. The TEACCH Approach to Autism Spectrum Disorders. Springer, New York, 2004.

16. Muller, M. J. Participatory design: the third space in HCI. Human-computer interaction: Development process (2003), 165-185.

17. Rasche, N., and Qian, C. Z. Work in progress: Application design on touch screen mobile computers (tsmc) to improve autism instruction. 2013 IEEE Frontiers in Education Conference (FIE) 0 (2012), 1-2.

18. Macedo, Isys ; Trevisan, Daniela G. ; CLUA, E. ; Vasconcelos, C. N. . Observed Interaction in Games for Down Syndrome Children. In: Annual Hawaii International Conference on System Sciences 2015 (HICSS 2015), 2015, Kauai. Proceedings of the 48th Annual Hawaii International Conference on System Sciences, 2015. v. 48. p. 320-327

19. Rodrigues, M. ASD-Teaching-Tool. Available : http://github.com/mrodrigues/ASDTeaching-Toolr, 2014.

20. Rodrigues, M. Devan-any-two. Available : https://github.com/mrodrigues/DEVAN-AnyTwo, 2014.

21. Romski, M. A., and Sevcik, R. A. Augmentative communication and early intervention: Myths and realities. Infants \& Young Children 18, 3 (2005), 174. 
22. Vermeeren, A. P. O. S., den Bouwmeester, K., Aasman, J., and de Ridder, H. Devan: A tool for detailed video analysis of user test data. Behaviour and Information Technology 21, 6 (2002), 403-423.

23. Williams, C., Wright, B., and Young, O. How to Live with Autism and Asperger Syndrome: Practical Strategies for Parents and Professionals. Jessica Kingsley Publishers, 2004.

24. Google Play. TalkinPictures. Available :

https://play.google.com/store/apps/details?id=com.androidinlondon.autismquicktalk\&hl=e n_US

25. meaVOX. Alternative communication for all. Available: http://meavox.com.br/

26. What's The Word Answer. Available: https://www.whatsthewordanswers.com

27. Appendix A. Available: https://github.com/thiagomalheiros/asdTools_Docs (only in Portuguese) 